Supplementary Material

\title{
Far-Infrared Synchrotron Spectroscopy of a Potentially Important Interstellar Isotopologue of Vinyl Alcohol: $\mathrm{CH}_{2} \mathrm{CHOD}$
}

\author{
Hayley Bunn ${ }^{2}$, Killian Hull ${ }^{1}$, Isaac Miller ${ }^{1}$, and Paul L. Raston ${ }^{1,2}$ \\ ${ }^{1}$ Department of Chemistry and Biochemistry, James Madison University, Harrisonburg, \\ Virginia 22807, USA \\ ${ }^{2}$ Department of Chemistry, University of Adelaide, SA 5005, Australia
}

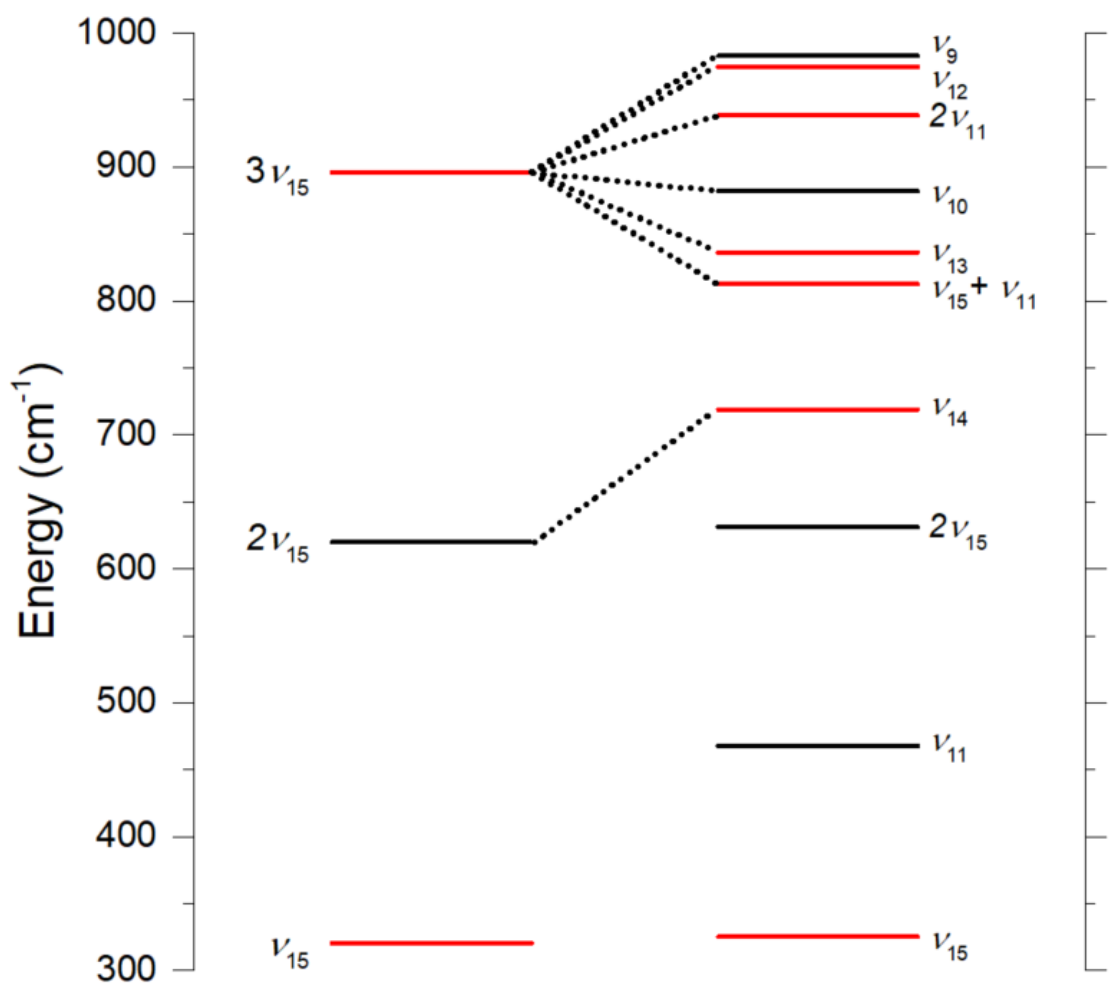

Figure S1. Experimental energy levels for the $v_{15} \mathrm{OD}$ torsional vibration of $s y n-V A-d_{1}$ (left) and the calculated states (with $\leq 2$ quanta of vibration) extracted from an anharmonic frequency calculation at the MP2/VQZ level, on a $\operatorname{CCSD}(T) / V Q Z$ optimised structure. The levels in red indicate asymmetric states and those in black, symmetric. The dotted lines indicate possible interactions with an energy difference of $<100 \mathrm{~cm}^{-1}$. 


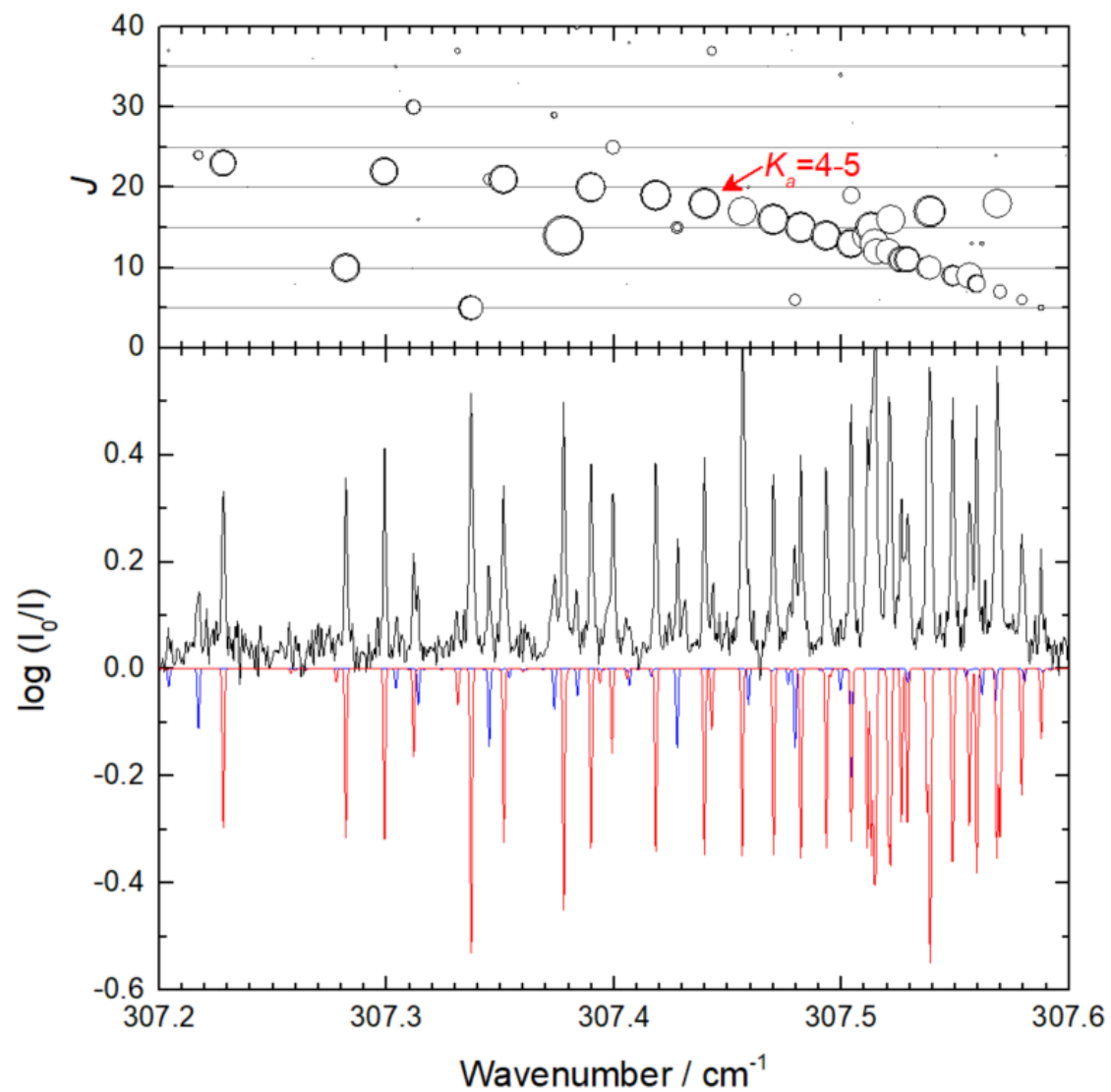

Figure S2. Same as Figure $4 \& 5$ (main text) but in the vicinity of the $K_{a}=4-5$ sub-band of the fundamental for syn-VA- $d_{1}$. This spectrum was collected under high flow-rate conditions. 


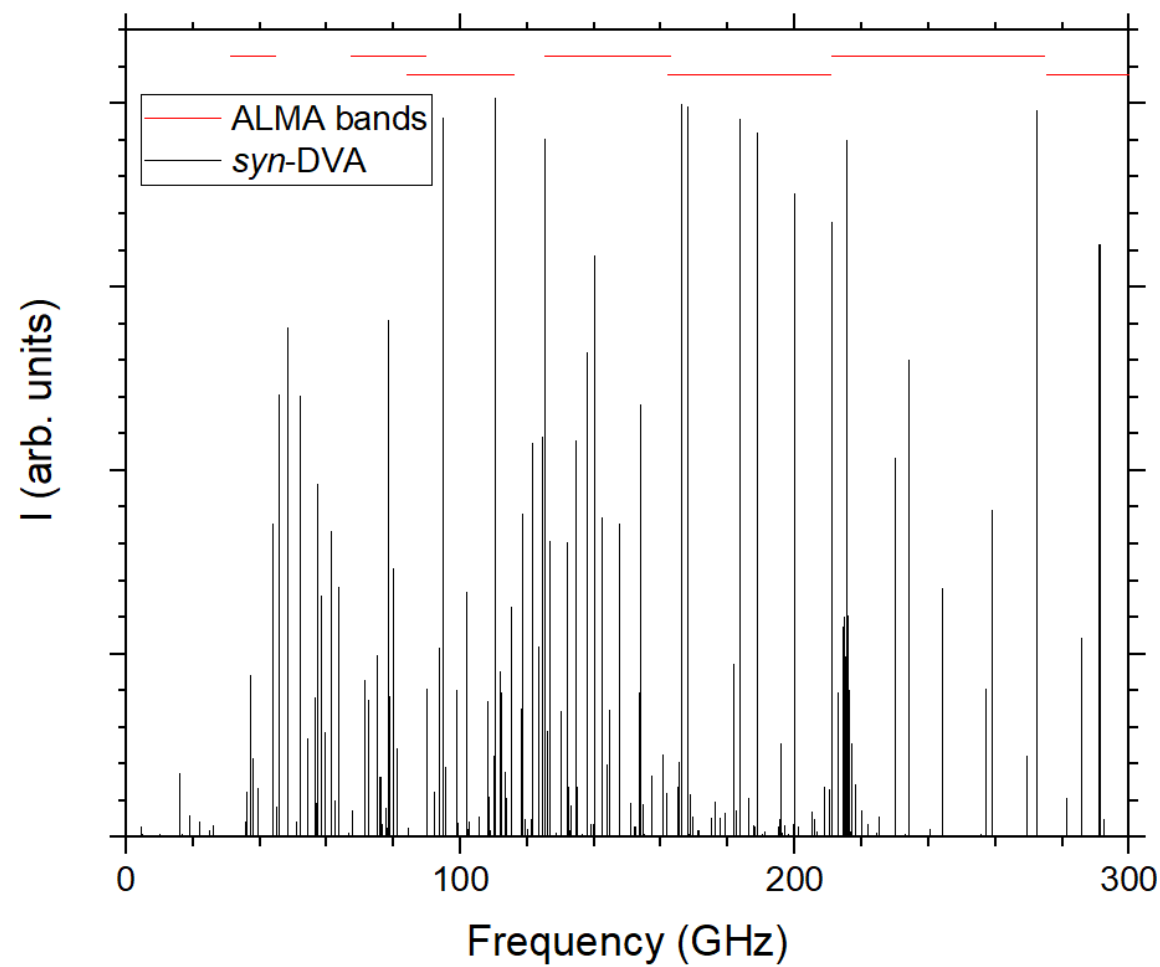

Figure S3. Simulated rotation spectrum of $s y n-\mathrm{VA}-d_{1}$ at $10 \mathrm{~K}$ using experimentally determined spectroscopic constants (including dipole moment components). Band coverage from the Atacama large millimetre/submillimetre array (ALMA) is indicated. 